\title{
Fatores de risco relacionados ao câncer de mama e a importância da detecção precoce para a saúde da mulher
}

\author{
Risk factors related to breast cancer and importance of early detection to woman health
}

Factores de riesgo relacionados con el câncer de mama y la importância de la detección temprana para la salude las mujeres

Laise Soares Costa ${ }^{1 *}$, Ana Luiza Oliveira do Carmo, Gustavo Gregorio Dias Firmiano', Júlia de Souza Silva Monteiro ${ }^{1}$, Luana Batista Faria ${ }^{1}$, Lindisley Ferreira Gomides ${ }^{1}$.

\section{RESUMO}

Objetivo: O presente estudo propôs reunir os fatores de riscos associados a neoplasia mamária e divulgar a detecção precoce como um importante método preventivo. Revisão bibliográfica: O Câncer de mama é um problema de saúde pública devido a natureza multifatorial e suas consequências epidemiológica, social e econômica. Apresenta alta taxa de incidência e gera transtornos psicológicos para as mulheres. Fatores como história reprodutiva, contraceptivos hormonais, ausência de filhos, primeira gravidez após 30 anos, menarca antes de 12 anos e menopausa após 55 anos foram os riscos mais apontados. A obesidade, o sedentarismo e o consumo de álcool também foram citados, além de fatores genéticos e hereditários. A prevenção primária (hábitos de vida saudável) e a secundária (rastreio) auxiliam no diagnóstico precoce, o qual favorece o tratamento e minimiza as taxas de morbimortalidade. Dentre essas têm-se o autoexame das mamas, o exame clínico e a mamografia, além de exames de imagem. Considerações finais: Faz-se necessária a conscientização sobre a importância da detecção precoce, através de palestras educativas junto aos gestores das saúdes pública e privada, equipe multidisciplinar e pacientes, divulgando propostas de monitoramento e acompanhamento a longo prazo, principalmente para as mulheres acima de 40 anos.

Palavras-chave: Câncer de mama, Detecção precoce, Fatores de risco.

\begin{abstract}
Objective: The present study proposed to gather risk factors associated with breast cancer and early detection as an important preventive method. Literature review: Breast cancer is a public health problem due to its multifactorial nature and its epidemiological, social and economic consequences. It has a high incidence rate and generates psychological disorders for women. Factors such as reproductive history, hormonal contraceptives, absence of children, first pregnancy after 30 years, menarche before 12 years of age and menopause after 55 years were the most mentioned risks. Obesity, sedentary lifestyle and alcohol consumption were also mentioned, in addition to genetic and hereditary factors. Primary prevention (healthy lifestyle habits) and secondary (screening) help in early diagnosis, which favors treatment and minimizes morbidity and mortality rates. These include breast self-examination, clinical examination and mammography, in addition to screening tests. Final considerations: It is necessary to raise awareness about the importance of early detection, through educational lectures with managers of public and private health, multidisciplinary team and patients, disclosing proposals for monitoring and long-term follow-up, especially for women above 40 years.
\end{abstract}

Key words: Breast cancer, Early detection, Risk factors.

\footnotetext{
${ }^{1}$ Faculdade Dinâmica do Vale do Piranga (FADIP), Ponte Nova - MG.

*E-mail: lalasocost@hotmail.com
} 


\section{RESUMEN}

Objetivo: Este estudio propuso recopilar factores de riesgo asociados con el cáncer de mama y divulgar la detección temprana como un método preventivo importante. Revisión de la literatura: El cáncer de mama es un problema de salud pública por su carácter multifactorial y sus consecuencias epidemiológicas, sociales y económicas. Tiene una alta tasa de incidencia y genera trastornos psicológicos para las mujeres. Factores como antecedentes reproductivos, anticonceptivos hormonales, ausencia de hijos, primer embarazo después de los 30 años, menarquia antes de los 12 años y menopausia después de los 55 años fueron los riesgos más mencionados. También se mencionaron la obesidad, el sedentarismo y el consumo de alcohol, además de factores genéticos y hereditarios. La prevención primaria (hábitos de vida saludables) y la secundaria (cribado) ayudan en el diagnóstico precoz, lo que favorece el tratamiento y minimiza las tasas de morbilidad y mortalidad. Estos incluyen el autoexamen de mamas, el examen clínico y la mamografía, además de las pruebas de imagen. Consideraciones finales: Haz-se necessário la concientizacion sobre la importancia da detección precoz, através de palestras educativas junto a los gestores de salud pública y privada, personal multidisciplinario y pacientes, divulgando propostas de monitoramento y seguimiento a longo plazo, principalmente para las mujeres acima de los 40 años.

Palabras clave: Cáncer de mama, Detección precoz, Factores de riesgo.

\section{INTRODUÇÃO}

O Câncer ( $C A$ ) é considerado, em nível nacional e mundial, um complexo problema de saúde pública devido a sua importância epidemiológica, social e econômica. A doença envolve fatores biológico-endócrinos, vida reprodutiva, comportamento e estilo de vida, sendo, portanto, de natureza heterogênea e multifatorial (BEZERRA HS, et al., 2018). A prevenção primária está diretamente relacionada ao controle dos fatores de risco, principalmente àqueles referentes ao estilo de vida e ao diagnóstico precoce através do rastreamento em pacientes com sinais e sintomas da doença (OHL IC, et al., 2016).

Dentre os tipos de CA, o de mama apresenta classificações que caracterizam seus aspectos celulares e a proliferação. O tumor benigno é descrito como uma neoplasia de proliferação lenta e células diferenciadas, enquanto o maligno é metastático, de proliferação abrupta e apresenta células indiferenciadas (BRITO MG, et al., 2019).

De acordo com Barcelos MR, et al. (2020), em 2012 foram notificados 1,6 milhão de novos casos de CA de mama no mundo, atingindo cerca de 324 mil óbitos, justificando $1^{\circ}$ lugar em incidência e mortalidade entre o público feminino. Pesquisas apontam que a doença atinge progressivamente um número maior de mulheres em faixas etárias mais baixas e apresenta taxas crescentes de mortalidade. Essa expressiva mortalidade ocorre não apenas devido à sua alta incidência, mas, também, ao fato de os casos serem descobertos tardiamente (TEIXEIRA LA e NETO LA, 2020).

Segundo dados do CONCORD-3 Allemani C, et al. (2018) as estimativas de sobrevida em cinco anos foram de 76,9\% (75,5 - 78) para o período de 2005 a 2009 e de 75,2\% (73,9 - 76,5) entre 2010 a 2014, correlacionando a falta de informações sobre a doença e, principalmente, dificuldades para o diagnóstico e tratamento adequados com os estágios mais avançados do CA de mama (INCA, 2019).

Além desses índices, o CA de mama tem sido considerado o mais temido entre as mulheres, principalmente pelos seus efeitos psicológicos, os quais afetam a percepção da sexualidade e a própria imagem pessoal. Em decorrência disso, é observado um forte impacto psicossocial, com mudanças no cotidiano, perdas emocionais e prejuízo na Qualidade de Vida (QV), expondo a vulnerabilidade gerada pela doença (VARGAS GS, et al., 2020).

Frente a esse cenário, as ações de conscientização e de controle do CA de mama no Brasil vêm sendo progressivamente incorporadas às Políticas Públicas de Saúde (PPS), com a perspectiva de elaborar uma maior organização da atenção à saúde em prol da garantia do acesso às informações e da continuidade do cuidado a população diagnosticada com a doença (INCA, 2019). 
Diante desse contexto, o presente trabalho buscou enfatizar os fatores de risco para o CA de mama e estabelecer discussões sobre a fisiopatologia da doença, contribuindo para a atualização acerca do tema. A proposta foi destacar a importância da detecção precoce da neoplasia mamária, bem como do acompanhamento longitudinal das pacientes com diagnóstico estabelecido, a fim de garantir o melhor prognóstico dessa condição patológica. Para tal, a presente revisão bibliográfica aborda os seguintes tópicos i) Fisiopatologia; ii) Fatores de risco; iii) Diagnóstico; iv) Tratamento; e v) Prevenção, os quais encontram-se dispostos na sequência abaixo.

\section{REVISÃO BIBLIOGRÁFICA}

\section{Fisiopatologia}

A fisiopatologia do CA de mama inicia-se pelo crescimento rápido e desordenado das células cancerígenas, com caráter agressivo e incontrolável, independente da exposição a agentes cancerígenos ou carcinógenos. Dessa forma, as células sofrem processo de mutação espontânea, as quais não alteram seu desenvolvimento normal. Porém, essas alterações podem ocorrer em genes especiais (proto-oncogenes), inativos em células normais, a princípio. Uma vez ativados, esses transformam-se em oncogenes, responsáveis pela malignização (cancerização) das células normais, tornando-as neoplásicas. As características individuais facilitam ou dificultam a instalação do dano celular (SANTOS TA e GONZAGA MF, 2018).

De uma forma geral, a formação do tumor (oncogênese ou carcinogênese) é lenta e pode levar vários anos para que uma célula cancerosa se prolifere e dê origem a um tumor visível, tempo determinado pela exposição a agentes cancerígenos ou carcinógenos, em uma dada frequência e período, bem como pela interação que desenvolvem entre si (SANTOS TA e GONZAGA MF, 2018). Os efeitos cumulativos desses diferentes agentes têm como resultado a origem das fases do CA: início, promoção, progressão e inibição do tumor. O período de latência varia de acordo com a intensidade do estímulo carcinogênico, a localização primária do CA e o potencial estímulo dos agentes envolvidos (INCA, 2020).

A neoplasia mamária localiza-se no quadrante superior externo. Geralmente as lesões são indolores, fixas e com bordas irregulares, associadas às alterações dermatológicas quando em estágio avançado (INCA, 2020). O tipo histológico invasor comumente observado no CA de mama é o carcinoma ducal invasivo (70\% a $80 \%$ dos casos), o qual possui origem nos ductos lactíferos, com rompimento da membrana basal e infiltração do tecido adjacente. A partir de então, pode ocorrer uma dispersão dessas células (metástase) para outras partes do corpo via sistema linfático e circulação sanguínea. O carcinoma lobular infiltrante é o segundo mais comum, com cerca de 5 a 15\% dos casos (PEREIRA HF, et al., 2017; ELICKER MA, et al., 2020).

A dificuldade de acesso ao diagnóstico e ao tratamento adequado, juntamente com a falta de informação sobre a doença e seus fatores associados, contribuem para que as pacientes procurem ajuda em estágios mais avançados do CA de mama, o que piora o prognóstico (GONÇALVES CV, et al., 2017; INCA, 2019).

Além da presença de nódulo na mama e/ou axila, dentre as principais manifestações clínicas do CA de mama podem ser citadas alterações de pele mamária, retrações com aspecto em casca de laranja ou abaulamentos e dor local (SANTOS TA e GONZAGA MF, 2018). Sintomas como ansiedade, insônia, depressão e estresse também já foram observados em decorrência do diagnóstico e do tratamento a longo prazo, reforçando a necessidade de um acompanhamento multidisciplinar no cuidado de pacientes oncológicos (PETEET J, et al., 1986).

\section{Fatores de risco}

Dentre os principais fatores de risco para o CA de mama podem ser citados idade avançada, a qual sinaliza uma longa exposição a fatores endógenos e exógenos durante a vida; características reprodutivas, tais como a menarca precoce, menopausa tardia, ausência de filhos, primeira gravidez após 30 anos e alterações hormonais. Além desses, a história familiar e pessoal, fatores genéticos e hereditários, bem como os hábitos de vida também são considerados (RIBEIRO PV, et al., 2021). 
Somando a esses, também é consensual na literatura hábitos não saudáveis como etilismo, tabagismo, sedentarismo, alimentação a base de produtos industrializados e, como consequência, a obesidade (SUNY, et al., 2017). O Quadro 1 reúne, de forma didática, os principais fatores de risco considerados na literatura.

Quadro 1 - Principais fatores de risco relacionados ao desenvolvimento de CA de mama.

\begin{tabular}{|l|}
\hline \multicolumn{1}{|c|}{ Fatores de risco relacionados ao CA de mama } \\
\hline Idade \\
\hline Vida reprodutiva da mulher \\
\hline Menarca antes dos 12 anos; \\
Menopausa tardia (após os 55 anos); \\
Primeira gestação acima de 30 anos; \\
Nuliparidade; \\
Uso frequente de anticoncepcionais orais*; \\
Alta densidade do tecido mamário; \\
Terapia de reposição hormonal após menopausa*; \\
Amamentação*. \\
\hline Histórico de doença mamária proliferativa benigna \\
\hline Hábitos de vida \\
\hline Obesidade*; \\
Uso excessivo de álcool (60 gramas por dia); \\
Alimentação não saudável; \\
Sedentarismo; \\
Tabagismo*. \\
\hline Influências ambientais \\
\hline Exposição à radiação ionizante entre a puberdade e 30 anos de idade. \\
\hline
\end{tabular}

Legenda: *Fatores que ainda divergem opiniões na literatura sobre a contribuição para o desenvolvimento de CA de Mama.

Fonte: Costa LS, et al., 2021; adaptado de Ministério da Saúde - Instituto Nacional do Câncer, 2011.

\section{Diagnóstico}

$\mathrm{Na}$ atualidade, o CA de mama é um dos desafios no cenário da saúde pública no Brasil, apontando a relação entre as taxas incidência e de mortalidade diretamente relacionadas a fatores que dificultam o acesso da população de risco aos serviços públicos de saúde, com pouco conhecimento sobre a doença juntamente a baixa realização dos métodos de rastreio e, consequentemente, um diagnóstico tardio em estágios mais avançados, o qual piora o prognóstico (GONÇALVES CV, et al., 2017). Por conseguinte, a estratégia inicial para a detecção precoce do CA de mama engloba diferentes ações de rastreamento como, por exemplo, a realização do Autoexame das Mamas (AEM), um exame no qual a própria mulher realiza a palpação e a inspeção das mamas seguindo orientações específicas da técnica (OLIVEIRA DA, et al., 2020).

O exame aborda diferentes recomendações conforme a idade e a existência ou não de casos de CA de mama na família. Logo, é valido salientar que, conforme as diretrizes para a detecção precoce do CA de mama no Brasil, a eficácia do rastreamento com o AEM não colabora com a redução da mortalidade global, uma vez que o exame não é capaz de descobrir tumores de até 1 centímetro, além de identificar lesões prémalignas ou ainda lesões muito pequenas, antes de se tornarem CA (SOCIEDADE BRASILEIRA DE MASTOLOGIA, 2017).

Somado a isso, o ato de se auto apalpar e não identificar nenhuma alteração faz com que as mulheres deixem de se preocupar e acabam não procurando atendimento médico para realização de exames de rastreamento com padrão ouro. Por isso, as falhas no rastreamento e a lentidão entre a confirmação e o tratamento contribuem para a mortalidade feminina. Posteriormente, o Exame Clínico das Mamas (ECM) é realizado por um profissional de saúde treinado, podendo ser um médico(a) ou enfermeiro(a). O ECM 
apresenta várias técnicas que são descritas na literatura médica, as quais incluem os componentes inspeção e palpação das mamas para verificação da existência de linfonodos (BARCELOS MR, et al., 2020).

Outro método importante é a Mamografia, a qual caracteriza-se como um exame de imagem básico e imprescindível para o diagnóstico das patologias mamárias, sendo o único reconhecido como técnica de rastreamento para o CA de mama nos seguimentos da saúde, além de ser o exame padrão ouro para lesões precursoras presentes na população de risco, consequentemente obtendo um diagnóstico precoce (INCA, 2021).

Recomenda-se que a mamografia de rotina, a cada dois anos, seja opção de rastreio para mulheres com idade entre 50 a 69 anos, sem sinais e sintomas de CA de mama. Nessa faixa etária é possível identificar as lesões mamarias em pacientes após a menopausa uma vez que, antes desse espaço de tempo, as mamas femininas se apresentam mais densas, reduzindo assim a sensibilidade do exame, com risco de resultados falso-negativos. Em casos especiais, nos quais são pontuados vários fatores de risco, o exame é recomendado a partir dos 35 anos de idade, com periodicidade anual (INCA, 2021).

Outra ferramenta utilizada no diagnóstico do CA de mama é a Ressonância Nuclear Magnética (RNM), método de imagem de escolha para pacientes que possuem mutações nos genes BRCA1 e BRCA2. Sua aplicação clínica é diretamente relacionada ao estadiamento de tumores, juntamente com a mamografia. A ultrassonografia também pode ser utilizada como método de rastreio adjuvante, tendo como foco principal, aquelas mulheres com diagnóstico anterior de tecido mamário denso que apresentam fatores de risco. A ultrassonografia é o único método usado isoladamente (BARCELOS MB, et al., 2020).

Diante do exposto, toda alteração suspeita de CA de mama, seja identificada em métodos de imagem ou até mesmo percebida inicialmente pela mulher, requer uma investigação mais aprofundada. Para tal, visando a busca pela padronização dos laudos mamográficos, foi adotado o modelo do sistema Breast Imaging Reporting and Data System (BI-RADS ${ }^{\mathrm{TM}}$ ), já utilizado pelo Colégio Americano de Radiologia. Esse sistema auxilia o médico quanto às condutas a serem tomadas a partir do laudo mamográfico. Ademais, é considerado o principal parâmetro para a padronização e a uniformização na prática da interpretação da mamografia, com a inclusão de seis categorias que descrevem a densidade radiológica do tecido mamário, as quais sofrem variações desde a mama quase inteiramente lipossubstituída até a mama extremamente densa (SILVA VJ, et al., 2019).

Em sua última edição, o BI-RADS ${ }^{\mathrm{TM}}$ passou a incluir, além da versão para mamografia, o mesmo princípio para classificação de exames de ultrassonografia e RNM. Assim, em linhas gerais, a classificação desse sistema abrange: A) Tipo 1 - mamas normais, sem achados mamográficos de malignidade; B) Tipo 2 - mamas moderadamente densas, achados mamográficos de benignidade; C) Tipo 3 - mamas heterogeneamente densas, achados mamográficos provavelmente benignos; D) Tipo 4 - mamas extremamente densas, achados mamográficos suspeito de malignidade (realizar biópsia); E) Tipo 5 - achados mamográficos altamente suspeitos de malignidade (realizar cirurgia e biópsia); e, por fim, F) Tipo 0 - resultado incompleto ou inconclusivo (FUNDAÇÃO OSWALDO CRUZ, 2018).

\section{Tratamento}

Graças ao desenvolvimento da ciência e da evolução tecnológica associada às pesquisas, a terapêutica para o CA de mama evoluiu nos últimos anos, principalmente no que diz respeito a cirurgias minimamente invasivas como, por exemplo, a busca pelo tratamento individualizado, adequado segundo o estadiamento da doença e as características biológicas do tumor, assim como as condições da paciente (idade, níveis séricos de hormônios, comorbidades e preferências) (PEREIRA AC, et al., 2018). Os tipos de tratamento do CA de mama são divididos em tratamento local, cirurgia, radioterapia (além de reconstrução mamária), tratamento sistêmico, quimioterapia, hormonioterapia e terapia biológica (INCA, 2020).

O prognóstico do CA de mama varia com a intensidade da doença, bem como das características do tumor. Quando a patologia é diagnosticada no início, o tratamento tem maior probabilidade curativa. Dessa forma, na presença de metástase, o objetivo do tratamento é conferir QV ao paciente, juntamente com aumento da sobrevida (INCA, 2020). Abaixo estão enumerados os estágios da doença, com suas respectivas características. 


\section{Estádios I e II}

Nessa fase, é importante a avaliação dos linfonodos axilares como direcionamento do prognóstico (GIULIANO A, et al., 2011). Em relação às mamas, o tratamento pode ser conservador (remoção apenas do tumor) ou com retirada da mama e subsequente reconstrução mamária, procedimento indicado quando a mastectomia é realizada (MORAN MS, et al., 2014).

Após a cirurgia, o tratamento com radioterapia pode ser iniciado. Ademais, o tratamento sistêmico será de acordo com o risco de reincidência (idade, complicação linfática, tamanho tumoral e grau de diferenciação), bem como as próprias características do tumor, com base na mensuração dos receptores hormonais (estrogênio e progesterona) e dos níveis de fator de crescimento epidérmico 2 (HER-2), com possível indicação de terapia biológica anti-HER-2 (HAMMOND ME, et al., 2010; WOLF AC, et al., 2013; INCA, 2020).

\section{Estádio III}

É possível observar o estágio III em tumores maiores, porém ainda localizados. Nesse caso, a quimioterapia é a modalidade terapêutica de escolha para início do tratamento sistêmico, na maioria das vezes. Observando o prognóstico do paciente, é possível também fazer associação e/ou inclusão de tratamento local, com radioterapia ou cirurgia, por exemplo (CORTAZAR P, et al., 2014; RODRIGUES JD, et al., 2015).

\section{Estádio IV}

No estágio IV, por sua vez, a escolha terapêutica busca o equilíbrio entre a resposta do tumor ao tratamento e o aumento da sobrevida, em avaliação aos efeitos colaterais em decorrência do próprio tratamento. Com isso, a equipe multidisciplinar pode acompanhar o paciente e observar se, de fato, está sendo alcançado um melhor suporte para a QV durante o enfrentamento da doença. O tratamento local é indicado apenas para casos restritos, sendo a terapia sistêmica a intervenção de escolha nesse estágio (CARDOSO F, et al., 2014; LOPES JV, et al., 2018).

\section{Prevenção: ferramentas e práticas}

Para a prevenção do CA de mama existem ferramentas e práticas que estão englobadas na seguinte classificação: A) prevenção primária e B) prevenção secundária. Na primeira, o paciente deve estar atento a questões simples do dia a dia que envolvem uma boa qualidade de vida, tais como hábitos de vida saudáveis; controle da obesidade, principalmente observando a variação da gordura da circunferência abdominal; prática de atividades físicas regulares e alimentação saudável (SANTOS SJ, et al., 2019).

A inflamação crônica induzida pelo excesso de lipídios em condições de sobrepeso e de obesidade pode promover a agressividade do CA e auxiliar na propagação do tumor, um processo mediado por citocinas inflamatórias. Com isso, a prática de exercícios físicos regulares e uma alimentação saudável - com mais produtos naturais e menos industrializados - são importantes fatores de prevenção (CORMANIQUE TF, et al., 2015).

Outro fator de risco de desenvolvimento de CA relacionado aos hábitos de vida é o tabagismo, o qual pode influenciar na progressão do CA de mama por atrapalhar o metabolismo hormonal. $O$ hábito de fumar tem sido associado a fatores prognósticos adversos, tais como metástases linfáticas e o maior risco de desenvolvimento de metástases no pulmão (SUN Y, et al., 2017).

Além do tabagismo, a ingestão de bebida alcoólica também representa um fator de risco considerável para o CA de mama, tanto em mulheres na pré-menopausa quanto na pós-menopausa (SOCIEDADE BRASILEIRA DE MASTOLOGIA, 2017). O etanol pode agir como carcinogênico, gerando aumento da permeabilidade da membrana celular a carcinógenos, inibição da detoxificação dos mesmos pelo fígado, com prejuízo do metabolismo de nutrientes e indução ao estresse oxidativo (SILVA SE, et al., 2013). Dessa forma, a ingestão de álcool sem moderação pode desencadear na ativação de um processo mutagênico e, com isso, elevar os níveis séricos de estrógenos, bem como a atividade de transcrição do receptor de estrógeno, aumentando, assim, a resposta da célula à ação desse hormônio (ROCHA ME, et al., 2020). 
Já a segunda classificação, a prevenção secundária, constitui-se pelo monitoramento através do ECM, realizado por médicos ou enfermeiros treinados, além do rastreamento, através da mamografia. Após os 40 anos de idade, toda mulher deve se submeter ao EC manualmente (SALDANHA RF, et al., 2019). Essas ações visam a obtenção de estratégias de conscientização, com a identificação de sinais e sintomas, juntamente com a confirmação diagnóstica precoce.

Vale ressaltar que, a remoção das barreiras para a diminuição da taxa de mortalidade por CA de mama na população feminina no Brasil compreende não apenas o fácil acesso a mamografia de rastreio, mas, também, o controle dos fatores preventivos, além da estruturação da rede assistencial, a qual oferece um rápido e oportuno meio de investigação diagnóstica e acesso humanizado ao tratamento de qualidade oferecido à paciente (INCA, 2019).

Sabe-se que, dentre os inúmeros desafios para que as mulheres estejam ativamente envolvidas com a prevenção, o acesso aos serviços de saúde ainda é considerado o maior entrave. Pesquisas apontam que quanto menor a escolaridade e a renda da família, maior é a dificuldade de acesso ao sistema de saúde, destacando os fatores sociodemográficos (escolaridade, renda familiar, vínculo empregatício) como agentes coadjuvantes de adesão ao rastreamento mamográfico e, consequentemente, a prevenção (OHL IC, et al., 2016).

\section{CONSIDERAÇÕES FINAIS}

Diante dos dados analisados foi possível observar que a detecção precoce do CA de mama e o cuidado com os hábitos de vida estão diretamente associados ao diagnóstico da doença, bem como ao seu prognóstico. Destaca-se a importância da realização da mamografia de rotina em mulheres com idade entre 50 a 69 anos, que não apresentam sinais e sintomas e, em casos especiais que incluem fatores de risco, em mulheres a partir dos 35 anos. $O$ conhecimento sobre os fatores de risco, prevenção, rastreamento e detecção precoce é de fundamental importância para o melhor acompanhamento médico e obtenção de resultados positivos no tratamento, com suporte no desenvolvimento de resiliência e na QV.

\section{REFERÊNCIAS}

1. ALLEMANI C, et al. Global surveillance of trends in cancer survival 2000-14 (CONCORD-3): analysis of individual records for 37513025 patients diagnosed with one of 18 cancers from 322 population-based registries in 71 countries. Lancet, 2018; 391(10125): 1023-1075.

2. BARCELOS MRB, et al. Diretrizes de rastreamento do câncer de mama com práticas personalizadas e baseadas em risco: estamos preparados? Femina, 2020; 48(11): 685-698.

3. BEZERRA HS, et al. Avaliação do acesso em mamografias no Brasil e indicadores socioeconômicos: um estudo espacial. Revista Gaúcha de Enfermagem, 2018; 39: e20180014.

4. BRITO MG, et al. Breast Cytology in Early Breast Cancer Detection: a review. Revista Espaço Ciência \& Saúde, 2019; 7(2): 20-34.

5. CARDOSO F, et al. ESO-ESMO 2nd International Consensus Guidelines for Advanced Breast Cancer (ABC2). Ann Oncol, 2014; 25(10): 1871-1888.

6. CORMANIQUE TF, et al. Estresse psicológico crônico e seu impacto no desenvolvimento de neoplasia mamária agressiva. Einstein, 2015; 13(3): 352-356.

7. CORTAZAR $P$, et al. Pathological complete response and long-term clinical benefit in breast cancer: the CTNeoBC pooled analysis. Lancet, 2014; 384(9938): 164-172.

8. ELICKER MA, et al. A citologia mamária na detecção precoce do câncer de mama: uma revisão. Revista Espaço Ciência \& Saúde, 2020; 7(2): 20-34.

9. FUNDAÇÃO OSWALDO CRUZ (FIOCRUZ). Sistema BI-RADS: Condutas. Portal de Boas Práticas em Saúde da Mulher, da Criança e do Adolescente, 2018.

10. GIULIANO AE, et al. Axillary dissection vs no axillary dissection in women with invasive breast cancer and sentinel node metastasis: A randomized clinical trial. Jama, 2011; 305: 569-575.

11. GONÇALVES CV, et al. O conhecimento das mulheres sobre a prevenção do câncer de mama. Ciência \& Saúde Coletiva, 2017; 22(12): 4073-4081.

12. HAMMOND ME, et al. American Society of Clinical Oncology/College Of American Pathologists guideline recommendations for immunohistochemical testing of estrogen and progesterone receptors in breast cancer. $\mathrm{J}$ Clin Oncol, 2010; 28: 2784-2795. 
13. INSTITUTO NACIONAL DE CÂNCER (BRASIL). A Situação do Câncer de Mama no Brasil. Rio de Janeiro: INCA, 2019.

14. INSTITUTO NACIONAL DE CÂNCER (BRASIL). Recomendações do Ministério da Saúde para o Rastreamento do Câncer de Mama. Rio de Janeiro: INCA, 2019.

15. INSTITUTO NACIONAL DE CÂNCER. ABC do Câncer: abordagens básicas para o controle do câncer. Rev. Atual. Rio de Janeiro: INCA, 2020.

16. INSTITUTO NACIONAL DE CÂNCER. Estimativa 2021. Parâmetros técnicos para rastreamento do câncer de mama. Rio de Janeiro: INCA, 2021.

17. INSTITUTO NACIONAL DE CÂNCER. Tratamento para o Câncer de Mama. Rio de Janeiro: INCA, 2020.

18. LOPES JV, et al. Impact of breast cancer and quality of life of women survivors. Rev Bras Enferm, 2018; 71(6): 30903096.

19. MORAN MS, et al. Society of Surgical Oncology-American Society for Radiation Oncology consensus guideline on margins for breast-conserving surgery with whole breast irradiation in stage I and II invasive breast cancer. Ann Surg Oncol, 2014; 21: 704-716.

20. OHL ICB, et al. Ações públicas para o controle do câncer de mama no Brasil: revisão integrativa. Revista Brasileira de Enfermagem, 2016; 69(4):793-803.

21. OLIVEIRA DA, et al. Autocuidado e prevenção do câncer de mama: conhecimento das estudantes de graduação em saúde. Rev. Acervo Saúde, 2020; (10): 1-8.

22. PEREIRA ACP, et al. Sistematização da assistência de enfermagem e o câncer de mama entre mulheres. Rev. Da ciência da saúde, 2018; 16(1): 39-47.

23. PEREIRA HFB, et al. Aspectos clínicos e patológicos do câncer de mama em mulheres jovens atendidas na FCecon entre 2003 e 2013. Revista Brasileira de Cancerologia, 2017; 63(2): 103-109.

24. PETEET J, et al. Pain characteristics and treatment in an outpatient cancer population. Cancer. 1986; 57(6): $1259-65$.

25. RIBEIRO PV, et al. Mutação RAD51D e o câncer de mama: relato de caso e achados na literatura. Rev. Acervo Científico, 2021; (19): 1-5.

26. ROCHA ME, et al. Câncer de mama: caracterização quanto a idade e aos aspectos tumorais (tipo de tumor e extensão). Brazilian Journal of Development, 2020; 6(1).

27. RODRIGUES JD, et al. An analysis of breast cancer prevention in Brazil. Ciência \& Saúde Coletiva, 2015; 20(10): 3163-3176.

28. SALDANHA RF, et al. Estudo de análise de rede do fluxo de pacientes câncer de mama no Brasil entre 2014 e 2016. Cadernos de Saúde Pública, 2019; 35(7): e00090918.

29. SANTOS TA, GONZAGA MFN. Fisiopatologia do câncer de mama e os fatores relacionados. Revista Saúde em Foco, 2018; 10:359-366.

30. SILVA SED, et al. As representações sociais do câncer de mama e no colo do útero no conhecimento da enfermagem brasileira. Revista eletrônica gestão e saúde, 2013; 4(3): 1130-45.

31. SILVA VJS, et al. BI-RADS Tumor Classification Through Image Minig. Symposium on Knowledge Discovery, Mining and Learning, KDMILE 2019.

32. SOCIEDADE BRASILEIRA DE MASTOLOGIA. Câncer de Mama. Documento científico - Consenso da Sociedade Brasileira de Mastologia - Regional Piauí, 2017.

33. SUN Y, et al. Risk factors and preventions of breast cancer. International Journal of Biological Sciences, 2017; 13(11): 1387-1397.

34. TEIXEIRA LA, NETO LAA. Breast cancer in Brazil: medicine and public health in 20th century. Saúde Soc. São Paulo, 2020; 29(3): e180753.

35. VARGAS GS, et al. Social support network of women with breast cancer. Revista online de pesquisa - Cuidado é fundamental, 2020; 12: 73-78.

36. WOLFF AC, et al. Recommendations for human epidermal growth factor receptor 2 testing in breast cancer: American Society of Clinical Oncology/College of American Pathologists clinical practice guideline update. J Clin Oncol, 2013; 31(31): 3997-4013. 